\title{
Phylogenetic analysis identifies the 'megabacterium' of birds as a novel anamorphic ascomycetous yeast, Macrorhabdus ornithogaster gen. nov., sp. nov.
}

\author{
Elizabeth K. Tomaszewski, ${ }^{1}$ Kathleen S. Logan, ${ }^{2}$ Karen F. Snowden, ${ }^{2}$ \\ Cletus P. Kurtzman ${ }^{3}$ and David N. Phalen ${ }^{1,2}$ \\ 1,2Department of Large Animal Medicine and Surgery ${ }^{1}$ and Department of Veterinary \\ Pathobiology ${ }^{2}$, College of Veterinary Medicine, Texas A\&M University, College Station, \\ TX 77843, USA
}

Correspondence

David N. Phalen

dphalen@cvm.tamu.edu
${ }^{3}$ Microbial Genomics and Bioprocessing Research Unit, National Center for Agricultural Utilization Research, USDA, Agricultural Research Service, Peoria, IL 61604-3999, USA
An organism commonly referred to as 'megabacterium' colonizes the gastric isthmus of many species of birds. It is weakly Gram-positive and periodic acid-Schiff-positive and stains with silver stains. Previous studies have shown that it has a nucleus and a cell wall similar to those seen in fungi. Calcofluor white M2R staining suggests that the cell wall contains chitin, a eukaryote-specific substance, and rRNA in situ hybridization demonstrates that it is a eukaryote. To characterize this organism phylogenetically, DNA was extracted from purified cells. rDNA was readily amplified by PCR with pan-fungal DNA primer sets and primer sets derived from the newly determined sequence, but not with bacteria-specific primer sets. Specific primer sets amplified rDNA from isthmus scrapings from an infected bird, but not from a non-infected bird or other control DNA. The sequence was confirmed to derive from the purified organism by in situ rRNA hybridization using a specific probe. Phylogenetic analysis of sequences of the 18S rDNA and domain D1/D2 of 26S rDNA showed the organism to be a previously undescribed anamorphic ascomycetous yeast representing a new genus. The name Macrorhabdus ornithogaster gen. nov., sp. nov. is proposed for this organism. The type material is CBS $9251^{\top}$ (=NRRL Y $-27487^{\top}$ ).
An organism commonly referred to as 'megabacterium' infects domestic birds (Huchzermeyer et al., 1993; Mutlu et al., 1997; Wieliczko \& Kuczkowski, 2000; Schulze \& Heidrich, 2001) as well as wild (Filippich et al., 1993; Pennycott et al., 1998) and companion birds (Dorrestein et al., 1980; Baker, 1992; Gerlach, 2001). These long, slender organisms $(2-3 \times 20-80 \mu \mathrm{m})$ stain with silver stains and periodic acid-Schiff (PAS) and are weakly Gram-positive. The 'megabacterium' is found in the isthmus between the glandular and grinding stomach, where it grows on the lumenal surface and may penetrate koilin (Dorrestein et al., 1980; van Herck et al., 1984). It is associated with a lymphoplasmacytic gastritis in poultry (Mutlu et al., 1997)

Published online ahead of print on 24 January 2003 as DOI 10.1099/ ijs.0.02514-0.

Abbreviations: D1/D2, domains 1 and 2; PAS, periodic acid-Schiff; PNA, peptide nucleic acid.

The GenBank/EMBL/DDBJ accession number for the partial 18S, ITS1, 5.8S, ITS2 and partial 26S rDNA sequence of Macrorhabdus ornithogaster is AF350243. and a chronic fatal wasting disease in companion birds (Gerlach, 2001).

The organism was originally thought to be a yeast because of its staining characteristics (Dorrestein et al., 1980). Subsequently, van Herck et al. (1984) concluded that it was a bacterium, as they were unable to demonstrate cytoplasmic organelles or a nucleus. They did, however, show nucleus-like structures in Geimsa-stained organisms, but interpreted them to be 'granules'. Scanlan \& Graham (1990) reported isolating a bacterium from the stomach of a budgerigar using standard microbiological techniques. The isolated bacterium, however, was smaller than the organism observed in vivo and was not characterized by PAS or silver stains. Attempts by other investigators to grow this organism with standard microbiological isolation techniques have been unsuccessful. However, Gerlach (2001) reported isolation of this organism on MRS medium, but was unable to maintain it past a few passages. Huchzemeyer et al. (1993) also reported isolating an organism from the proventriculus of ostriches using MRS agar. This organism had the same biochemical properties as the one isolated by 
Scanlan \& Graham (1990), but was smaller than those seen histologically, and its ability to stain with PAS and silver stains was not reported.

More recent work suggested that the 'megabacterium' is, in fact, a yeast. In vivo trials showed that the 'megabacterium' was susceptible to amphotericin B, but not to antibacterial antibiotics (Filippich \& Perry, 1993). It stained strongly with calcofluor white M2R (Moore et al., 2001) and blancophor BA (Ravelhofer et al., 1998), stains that bind chitin, a polysaccharide not found in bacteria (Monheit et al., 1984). A nucleus was demonstrated by electron microscopy and in situ hybridization with a pan-eukaryote rRNA probe was positive (Ravelhofer-Rotheneder et al., 2000).

In this study, budgerigars naturally infected with the 'megabacterium' were killed humanely and longitudinal strips of the stomach containing the distal proventriculus, isthmus and proximal ventriculus were fixed in buffered formalin and paraffin-embedded. Thin sections were stained with haematoxylin and eosin, PAS, methenamine silver, Brown and Brenn and calcofluor white M2R (Monheit et al., 1984). Organisms obtained from scrapings of the remaining gastric isthmus were heat- or methanolfixed and stained with PAS, methenamine silver, Gram stain and calcofluor white MR2. Additional organisms were acid-digested and Giemsa-stained.

Aliquots of the organisms were washed several times in sterile PBS and purified by layering onto a $10-40 \%(\mathrm{w} / \mathrm{v})$ sucrose gradient which was centrifuged for $1 \mathrm{~h}$ at $14600 \mathrm{~g}$. The pellet was washed and suspended in PBS. Wet mounts of this suspension revealed no other organisms or debris. Genomic DNA was isolated from the suspension using mechanical disruption and a Puregene DNA isolation kit. Amplification and sequencing of ITS and 26S fungal ribosomal genes was done using previously described panfungal primers (White et al., 1990; Sandhu et al., 1995). New primers were designed to amplify and sequence the $18 \mathrm{~S}$ rDNA. To this end, the sequence of Saccharomyces cerevisiae 18 SDNA (GenBank accession no. J01353) was compared with that of other yeasts. Primers were then selected from conserved sequences found to be present in most or all yeasts compared. The forward primers were Sm1 (ATCTGGTTGATCCTGCCAGTAGTC; positions 2-25), BIG 1 (AGTGAAACTGCGAATGGCTC; 80-99), Sm3 (CTGAGAAACGGCTACCACATCC; 394-415) and Sm5 (AACTACTGCGAAAGCATTTGCC; 928-949). The reverse primers were Sm2 (CAATACGCCTGCTTTGAACACTC; 761-783), Sm4 (CTTCGATCCCCTAACTTTCGTTC; 971-993) and Sm6 (CACCTACGGAAACCTTGTTACGAC; 1756-1778). All primers are written in the $5^{\prime}-3^{\prime}$ direction. Positions refer to the $S$. cerevisiae rDNA sequence. Using these primers, DNA was routinely amplified by PCR, purified by gel electrophoresis and sequenced (Tomaszewski et al., 2001). A $3004 \mathrm{bp}$ sequence was obtained that contained the majority of the $18 \mathrm{~S}$, the entire ITS and the $5^{\prime}$ portion of the $26 \mathrm{~S}$ rDNA.
To verify that the amplified sequence was from a single organism, primers BIG 1 and Sm6 and ITS 5 (White et al., 1990) and U2 (Sandhu et al., 1995) were used to produce two large overlapping amplicons. A sequence from the $18 \mathrm{~S}$ rDNA of the organism but not found in any reported fungal sequence was included in the overlap. The two amplicons were inserted into vectors and cloned in competent cells. Plasmid inserts from selected recombinant colonies were sequenced with universal primers T7pl, M13 and the newly developed primers.

A PCR primer (AGY1) (GGACTTATATTACTAGTCAGATGG; positions 620-643 in the rDNA of the organism) that did not match reported sequences of other known fungi was developed from the $18 \mathrm{~S}$ rDNA. It was used in combination with $\mathrm{Sm} 2$ to show the specificity of the determined sequence. DNA extracted from scrapings of the isthmus of a naturally infected budgerigar, a budgerigar without infection, Candida albicans and a Lactobacillus sp. was subjected to PCR with these primers. Bacteria-specific PCR primers (Relman, 1993) were also used in a PCR with DNA from the purified organism to show that it was not a bacterium. DNA from Lactobacillus sp. was used as a positive control.

In situ hybridization with a commercially synthesized peptide nucleic acid (PNA) probe (AATTGAACCAGGACG; positions 704-718 in the rDNA of the organism) (Applied Biosystems) that targeted an rRNA sequence not found in other previously reported sequences of fungi was performed as described by Oliveira et al. (2001) with minor modifications. Isthmus mucosa scrapings collected from infected budgerigars were mixed with $C$. albicans and placed in wells of Teflon-coated slides and heat-fixed at $80^{\circ} \mathrm{C}$ for $1 \mathrm{~h}$. Wells were then covered with approximately $20 \mu \mathrm{l}$ hybridization solution containing $10 \%(\mathrm{w} / \mathrm{v})$ dextran sulfate, $10 \mathrm{mM} \mathrm{NaCl}, 20 \%$ formamide, $0 \cdot 1 \%(\mathrm{w} / \mathrm{v})$ sodium pyrophosphate, $0 \cdot 2 \%(\mathrm{w} / \mathrm{v})$ polyvinylpyrrolidone, $0 \cdot 2 \%$ (w/v) Ficoll, 5 nM disodium EDTA, $1 \%$ (v/v) Triton X-100, $50 \mathrm{mM}$ Tris/ $\mathrm{HCl}$ and $1 \mathrm{pM}$ PNA probe, overlaid with a cover slip and incubated for $30 \mathrm{~min}$ at $55^{\circ} \mathrm{C}$. The cover slips were removed by immersion in PBS plus $0 \cdot 1 \%$ Tween (PBST) for 1-2 min at room temperature. Slides were washed in preheated PBST $\left(59-69^{\circ} \mathrm{C}\right)$ for $30 \mathrm{~min}$, rinsed with PBS and air-dried. Each smear was mounted with Slow Fade light antifade kit (Molecular Probes) and a cover slip. The stained cells were visualized with a fluorescence microscope and photographed.

Initial placement of the bird pathogen among the ascomycetous yeasts resulted from a BLAST search of $18 \mathrm{~S}$ rDNA sequences maintained in GenBank. Following this, phylogenetic relationships were evaluated further from comparison of domains 1 and 2 (D1/D2) of 26S rDNA with sequences from all currently known ascomycetous yeasts (Kurtzman \& Robnett, 1998; and subsequent GenBank entries). The dataset was analysed by maximum parsimony as well as by neighbour joining with the Kimura twoparameter correction using PAUP 4.03a (Swofford, 1998). 
Sequence data were aligned visually with Qedit 2.15 (SemWare) and regions of uncertain alignment, including indels, were removed from the dataset before analysis. The dataset comprised 487 characters, of which 219 characters were parsimony-informative.

Haematoxylin and eosin-stained sections of the isthmus demonstrated numerous densely packed, long, filamentous organisms that were identical in size $(2-3 \mu \mathrm{m}$ wide and 20-80 $\mu \mathrm{m}$ long), shape and staining characteristics to the so-called megabacterium. The organisms were PAS- and methenamine silver-positive but were only weakly Grampositive. Organisms from scrapings were of the same dimensions (Fig. 1a) and staining characteristics. Gramstained preparations showed that organisms longer than $20 \mu \mathrm{m}$ were, in fact, chains of organisms each separated by a transverse septum (Fig. 1c). The number of cells ranged from one to four, with two and three being most common. Small, probably new, growing cells were often seen at either end of a chain of cells. Acid-digested and Giemsa-stained organisms contained one to four evenly spaced, dense, elongate and oval basophilic structures (Fig. 1b).

The sequence determined by sequencing the overlapping clones was identical to that obtained by sequencing the original smaller overlapping PCR products. Comparison of this sequence with those in GenBank showed that, as expected, it contained the majority of the SSU rDNA, the entire $5 \cdot 8 \mathrm{~S}$, ITS regions and $697 \mathrm{bp}$ of the $5^{\prime}$ end of the LSU rDNA. The sequence obtained was verified to be from the organism seen histologically by PCR with specific primers and in situ hybridization with a specific PNA probe. The specific PCR primer set (AGY1/SM2) only amplified DNA from the isthmus scraping from the infected budgerigar and not from an isthmus scraping from a non-infected budgerigar or from C. albicans or Lactobacillus DNA. The sequence of the amplified product was identical to the expected sequence. DNA from Lactobacillus was amplified with the bacteria-specific primer pairs, but these primers did not produce an amplicon from DNA of the organism purified from isthmus scrapings nor from C. albicans DNA. The organisms from isthmus scrapings hybridized with the rRNA PNA probe, but $C$. albicans did not (Fig. 2a, b).

A comparison of the $18 \mathrm{~S}$ rDNA sequence with those in GenBank placed the budgerigar fungus well within the ascomycetous yeast clade but, because relatively few $18 \mathrm{~S}$ sequences are available for yeasts, only broad relationships were discerned. Further analysis was made from the D1/ D2 domain of $26 \mathrm{~S}$ rDNA, because these sequences are available for all currently recognized ascomycetous yeasts (Kurtzman \& Robnett, 1998; and subsequent GenBank entries). Phylogenetic trees from both analyses were concordant and strongly supported placement of the budgerigar fungus within the ascomycetous yeast clade (bootstrap value $97 \%$ ), as well as showing it to be a unique species. However, neither gene sequence provides strong internal branch support and the location of the budgerigar
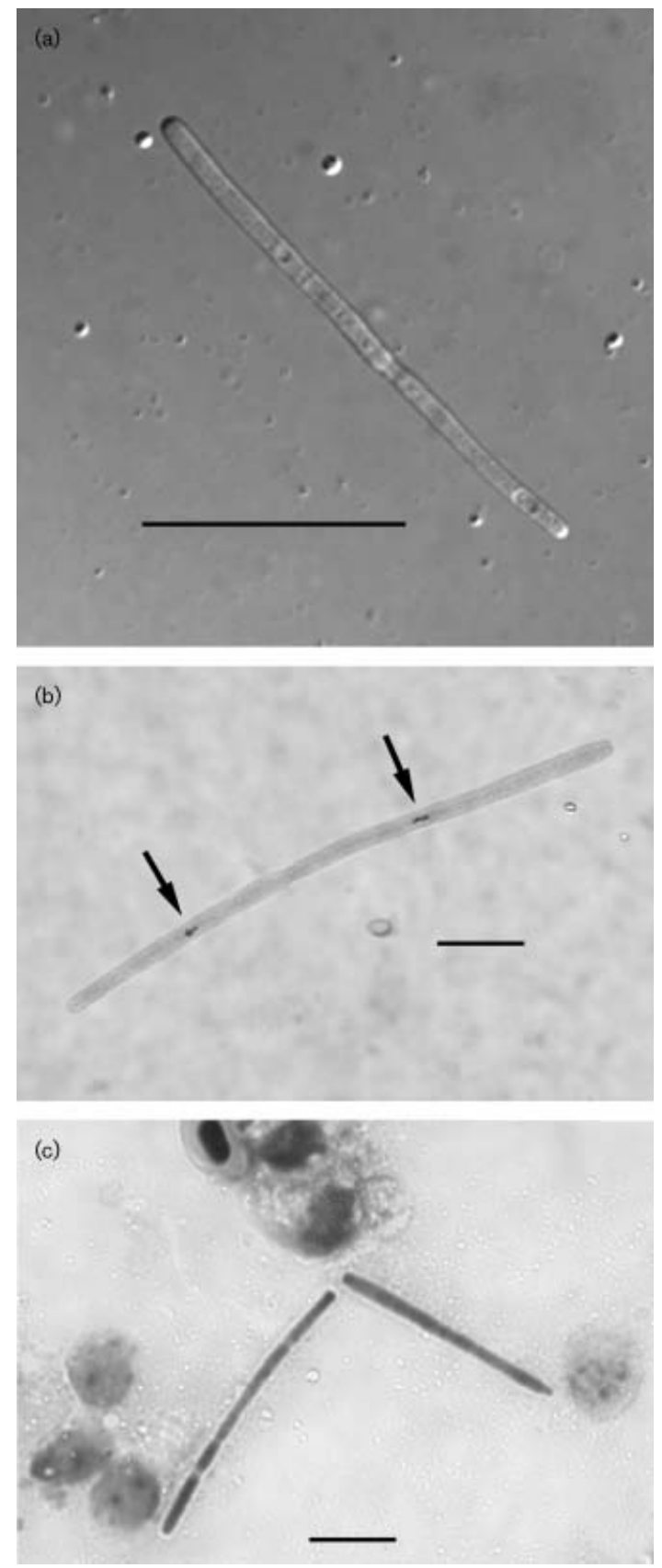

Fig. 1. Micrographs of Macrorhabdus ornithogaster gen. nov., sp. nov. in scrapings of the isthmus from a budgerigar: wet mount (a), acid-digested and Geimsa-stained (b) and Gram-stained (c). Structures believed to be nuclei are seen in the Geimsastained organism (b, arrows). Bars, $30 \mu \mathrm{m}$ (a) and $10 \mu \mathrm{m}$ (b, c).

yeast within the ascomycetous yeast clade is only approximate from these datasets. The 26S D1/D2 analysis suggests the budgerigar yeast to be weakly associated with and basal to the Dipodascus and Metschnikowia clades, as shown from its placement among representative species in Fig. 3. Further phylogenetic resolution will require analysis of multiple gene sequences. 


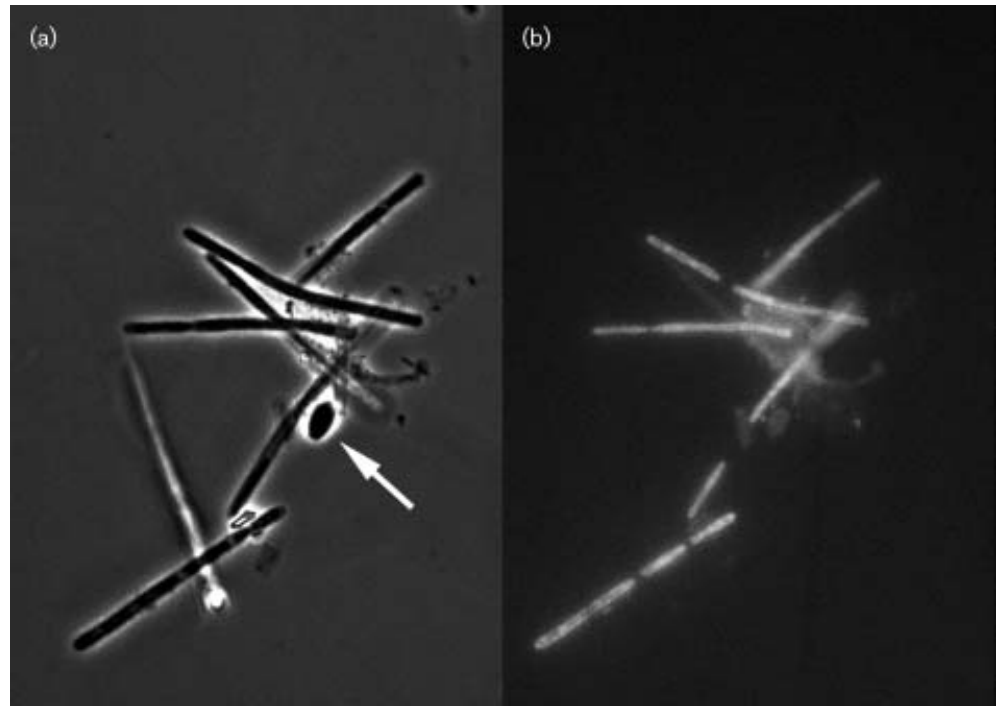

Fig. 2. Bright-field (a) and PNA fluorescence in situ hybridization (b) images of cells of $M$. ornithogaster gen. nov., sp. nov. and C. albicans (arrow). The M. ornithogasterspecific probe for rRNA is selectively localized in the cytoplasm of $M$. ornithogaster cells.

Our rDNA sequence findings are consistent with those of previous investigators, who showed by in situ hybridization that the organism contained eukaryote rRNA and a nucleus (Ravelhofer-Rotheneder et al., 2000). These findings are also consistent with the observations that this organism stains with both calcofluor MR2 and blancophor BA, stains that detect chitin, a molecule that is only found in eukaryotes. Given that this organism is a yeast, we conclude that the regularly spaced 'granules', first observed by van Herck et al. (1984), are, in fact, nuclei.

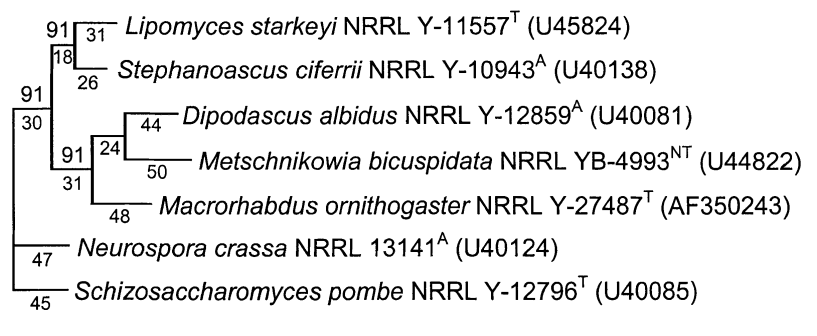

Fig. 3. Phylogenetic tree with selected representative taxa showing the placement of $M$. ornithogaster gen. nov., sp. nov. near the Dipodascus and Metschnikowia clades, as represented by one of two most-parsimonious tress derived from maximum-parsimony analysis of LSU rDNA domain D1/D2. Branch lengths, given below each branch, are proportional to nucleotide differences. Numbers above nodes are percentage frequencies with which a given branch appeared in 1000 bootstrap replicates. Frequencies under $50 \%$ are not given. Tree length, 394; consistency index, 0.807; retention index, 0.424; rescaled consistency index, 0.342; homoplasy index, 0.193. Each of the species shown is represented by the type strain or an authentic strain when no living type material was available. GenBank accession numbers for reference taxa are those given by Kurtzman \& Robnett (1998). Schizosaccharomyces pombe was the outgroup species in the analysis. T, Type strain; NT, neotype strain; A, authentic strain.
Previously, some investigators have concluded that the socalled megabacterium could be cultured from the ventriculus by using standard microbiological techniques and is, in fact, a bacterium (Scanlan \& Graham, 1990). The potential pitfall associated with culturing a lesion is that an organism that grows readily under the culture conditions, but is present in relatively small numbers, can appear to be the only organism present if the common organism in the lesion does not grow under these same conditions. It is therefore incumbent on investigators to prove that the organism that they have cultured is the same one that was seen in situ. The descriptions of bacteria, believed to be the 'megabacterium', that have been isolated from the gastric mucosa of birds have not been rigorous. Silver, PAS and calcofluor white M2R staining were not reported. Additionally, in two instances, the isolated organism was considerably smaller than the organisms seen in situ (Scalan \& Graham, 1990; Huchzermeyer et al., 1993). Based on our findings, we conclude that the bacteria isolated by these investigators represent either permanent or transient flora of the stomach of the birds they cultured, but are not the PAS-positive, silver-staining and calcofluor-positive organism that we have shown to be an ascomycetous yeast.

\section{Latin diagnosis of Macrorhabdus Tomaszewski, Logan, Snowden, Kurtzman \& Phalen gen. nov.}

Cellulae vegetativae elongatae, fissione divisae, singulae aut brevi catena compositae. Ascosporae non fiunt. Parasitus avium. Species typicam, Macrorhabdus ornithogaster.

\section{Description of Macrorhabdus Tomaszewski, Logan, Snowden, Kurtzman \& Phalen gen. nov.}

Macrorhabdus (Mac.ro.rhab' dus. Gr. masc. adj. macro from macros long; N.L. masc. n. rhabdus from Gr. masc. n. rabdos rod; N.L. n. Macrorhabdus long rod).

Vegetative cells are elongate, divide by fission and are single 
or in short chains. Ascospores are not formed. The organism is parasitic to birds. The type species is Macrorhabdus ornithogaster.

\section{Latin diagnosis of Macrorhabdus ornithogaster Tomaszewski, Logan, Snowden, Kurtzman \& Phalen sp. nov.}

Cellulae vegetativae elongatae $(2-3 \times 8-20 \mu \mathrm{m})$, fissione divisae. Cellulae singulae aut brevi seri usque et cellulas quattuor catenatae. Ascosporae non funt. Parasitus avium. Typus: NRRL Y-27487 ${ }^{\mathrm{T}}$, designat stirpem typicam. Isolata a Melopsittacus undulatus, College Station, TX, USA, depositata in collectione culturarum ARS (NRRL), Peoria, IL, USA.

\section{Description of Macrorhabdus ornithogaster Tomaszewski, Logan, Snowden, Kurtzman \& Phalen sp. nov.}

Macrorhabdus ornithogaster (or.ni.tho.gas'ter. Gr. gen. fem. or masc. n. ornitho from ornis bird; Gr. fem. n. gaster stomach; N.L. gen. n. ornithogaster of the stomach of a bird).

Vegetative cells are elongate $(2-3 \times 8-20 \mu \mathrm{m})$ and divide by fission. Cells are single or in short chains of two to four cells. Ascospores are not formed. The species is parasitic to birds and is found in gastric tissue. The type material was excised from an infected budgerigar in College Station, TX, USA, and consists of infected preserved tissue that is deposited at the Centraalbureau voor Schimmelcultures, Utrecht, The Netherlands, as CBS $9251^{\mathrm{T}}$ and the Agriculture Research Service Culture Collection (NRRL), Peoria, IL, USA as NRRL Y-27487 ${ }^{\mathrm{T}}$. This is the type specimen for the genus Macrorhabdus.

\section{Acknowledgements}

We thank Lucio Filippich, Nancy Keller, Pat Holman and John Roths for technical assistance and Brian Berridge for supplying the bacterial PCR primers. We also thank Jason Millership, Laurie Jaeger and Larry Wadsworth for excellent photographic assistance, Christie J. Robnett for rDNA database compilation, Christoph F. Konrad for providing the Latin descriptions and Susan W. Phalen for her assistance with the Greek derivations. This project was supported by the Schubot Exotic Bird Health Center, Texas A\&M University, Central California Avian Society, the Georgia Cage Bird Club, the International Parrotlet Society and the Peninsula Caged Birds Society.

\section{References}

Baker, J. R. (1992). Megabacteriosis in exhibition budgerigars. Vet $\operatorname{Rec} 131,12-14$.

Dorrestein, G. M., Zwart, P. \& Buitelaar, M. N. (1980). Problems arising from disease during the periods of breeding and rearing canaries and other aviary birds. Tijdschr Diegeneesk 105, 535-543.

Filippich, L. J. \& Perry, R. A. (1993). Drug trials against Megabacteria in budgerigars (Melopsittacus undulatus). Aust Vet Pract 23, 184-189.

Filippich, L. J., Boyle, D. A., Webb, R. \& Fuerst, J. A. (1993). Megabacteria in birds in Australia. Aust Vet Pract 23, 71-76.
Gerlach, H. (2001). Megabacteriosis. Semin Avian Exotic Pet Med 10, 12-19.

Huchzermeyer, F. W., Henton, M. M. \& Keffen, R. H. (1993). High mortality associated with megabacteriosis of proventriculus and gizzard in ostrich chicks. Vet $\operatorname{Rec} 133,143-144$.

Kurtzman, C. P. \& Robnett, C. J. (1998). Identification and phylogeny of ascomycetous yeasts from analysis of nuclear large subunit (26S) ribosomal DNA partial sequences. Antonie van Leeuwenhoek 73, 331-371.

Monheit, J. E., Cowan, D. F. \& Moore, D. G. (1984). Rapid detection of fungi in tissues using calcofluor white and fluorescence microscopy. Arch Pathol Lab Med 108, 616-618.

Moore, R. P., Snowden, K. F. \& Phalen, D. N. (2001). A method of preventing transmission of so-called 'megabacteria' in budgerigars (Melopsittacus undulatus). J Avian Med Surg 15, 283-287.

Mutlu, O. F., Seckin, S., Ravelhofer, K., Hildebrand, R.-A. \& Grimm, F. (1997). Proventriculitis in domestic fowl (Gallus gallus var. dom. L., 1758) caused by megabacteria. Tierärztl Prax 25, 460-462.

Oliveira, K., Haase, G., Kurtzman, C., Hyldig-Nielsen, J. J. \& Stender, H. (2001). Differentiation of Candida albicans and Candida dubliniensis by fluorescent in situ hybridization with peptide nucleic acid probes. J Clin Microbiol 39, 4138-4141.

Pennycott, T. W., Ross, H. M., McLaren, I. M., Park, A., Hopkins, G. F. \& Foster, G. (1998). Causes of death of wild birds of the family Fringillidae in Britain. Vet Rec 143, 155-158.

Ravelhofer, K., Rotheneder, R., Gareis, M., Suttner, R., Wolf, O., Matiello, R. \& Kösters, J. (1998). Megabakteriosen bei verschiedenen Vogelspezies. In DVG Tagung über Vogelkrankheiten, vol. 11, pp. 95-104. Gießen: Deutsche Veterinärmedizinische Gesellschaft.

Ravelhofer-Rotheneder, K., Engelhardt, H., Wolf, O., Amann, R., Breuer, W. \& Kösters, J. (2000). Taxonomic classification of "megabacteria" isolates originating from budgerigars (Melopsittacus undulatus Shaw, 1805). Tierärztl Prax 28, 415-420.

Relman, D. A. (1993). The identification of uncultured microbial pathogens. J Infect Dis 168, 1-8.

Sandhu, G. S., Kline, B. C., Stockman, L. \& Roberts, G. D. (1995). Molecular probes for diagnosis of fungal infections. J Clin Microbiol 33, 2913-2919.

Scanlan, C. M. \& Graham, D. L. (1990). Characterization of a Gram-positive bacterium from the proventriculus of budgerigars (Melopsittacus undulatus). Avian Dis 34, 779-786.

Schulze, C. \& Heidrich, R. (2001). Megabakterien-assoziierte proventridulitis beim nutzgefügel in Brandenburg. Dtsch Tieräztl Wochenschr 108, 264-266.

Swofford, D. L. (1998). PAUP* 4.0: phylogenetic analysis using parsimony. Sunderland, MA: Sinauer Associates.

Tomaszewski, E., Wilson, V. G., Wigle, W. L. \& Phalen, D. N. (2001). Detection and heterogeneity of herpesviruses causing Pacheco's disease in parrots. J Clin Microbiol 39, 533-538.

van Herck, H., Duijser, T., Zwart, P., Dorrestein, G. M., Buitelaar, M. \& van der Hage, M. H. (1984). A bacterial proventriculitis of canaries. Avian Pathol 13, 561-572.

White, T. J., Bruns, T., Lee, S. \& Taylor, J. (1990). Amplification and direct sequencing of fungal ribosomal RNA genes for phylogenetics. In PCR Protocols: a Guide to Methods and Applications, pp. 315-322. Philadelphia: Academic Press.

Wieliczko, A. \& Kuczkowski, M. (2000). Selected issues of infectious diseases in ostrich (Struthio camelus). Med Weter 56, 23-28. 